\title{
Comparative analysis of sensory activity of carbon nanotubes with boundary modification
}

\author{
Natalia Boroznina ${ }^{1}$, Irina Zaporotskova ${ }^{1}$, Sergei Boroznin $^{1}$, Lev Kozhitov $^{2}$, Pavel Zaporotskov $^{1}$ \\ ${ }^{1}$ Volgograd State University \\ prospect Universitetskii, 100, 400062, Volgograd, Russia \\ boroznina.natalya@volsu.ru; irinazaporotskova@gmail.com \\ ${ }^{2}$ NUST MISIS \\ Leninsky Prospekt, 4, 119049, Moscow, Russia \\ kozitov@misis.ru
}

\begin{abstract}
The high sensitivity of the electronic properties of nanotubes to molecules adsorbed on their surface and the unparalleled unit surface providing for this high sensitivity make CNT a promising starting material for the development of superminiaturized chemical and biological sensors. Sorption gas sensors are the largest group of gas sensors. Their main operation principle is adsorption during which an adsorbed gas molecule transfers an electron to or takes it from a nanotube. In this paper we represent the investigation of sensor selectivity for alkali metals and their ions for boundary modified with carboxyl, amino or nitro group carbon nanotube. It has been found that sensors fabricated on their basis will have high selectivity and response to the presence of ultralow quantities of materials, e.g. metals included in salts and alkali, and this shows good promise for their use in chemistry, biology, medicine etc.
\end{abstract}

Keywords: Carbon nanotubes, nanosensors, adsorption, quantum-chemical calculations, density functional theory, boundary modification, carboxyl group.

\section{Introduction}

The discovery of carbon nanotubes is one of the most important achievements of the advanced science. Nanotubes are the new materials with unique physicochemical properties. Carbon nanotubes can find applications in a great number of areas [1,2]: as additives to polymers, in autoelectron emission for cathode rays of lighting components, as elements of flat displays and lithium battery anodes. It may be use as hydrogen storage, composite materials, nanoprobes, haigly-sensitive sensors, ect.

A nanotube is a surface structure, so its whole weight is concentrated in its surface layers. This feature is the origin of the uniquely large unit surface of tubulenes which in turn predetermines their electrochemical and adsorption properties [3]. The high sensitivity of the electronic properties of nanotubes to molecules adsorbed on their surface and the unparalleled unit surface providing for this high sensitivity make CNT a promising starting material for the development of superminiaturized chemical and biological sensors. The operation principle of these sensors is based on changes in the V-I curves of nanotubes as a result of adsorption of specific molecules on their surface. The use of CNT in sensor devices is one of their most promising applications in electronics. Several types of gas sensors on the basis of the CNT are discussed in literature [4]. It include sorption gas sensors, ionization gas sensors, capacitance gas sensors, resonance frequency shift gas sensors. Experimental and theoretical studies showed that CNT are an extremely promising material for further use in the field. Further development of nanotube technologies will provide new physical objects the properties of which will be of great scientific and practical interest. Thanks to their unique structure and properties the CNT can be used as active elements of sensors for the detection of numerous materials including gases, organic compounds, metals etc. Sensors fabricated on their basis will have high selectivity and response to the presence of ultralow quantities of materials, e.g. metals included in salts and alkali, and this shows good promise for their use in chemistry, biology, medicine etc.

The main operation principle of sorption gas sensors is adsorption during which an adsorbed gas molecule transfers an electron to or takes it from a nanotube. This changes the electrical properties of the CNT, and this change can be detected [3]. There are gas sensors based on pure CNT, on CNT modified by functional groups, metals, polymers or surface defects. 
CNT modification changes the electronic properties of the nanotubes and increases their selectivity and response to specific gases.

In [5] reported that a sensor was designed for detecting gases and organic vapors at room temperature the detection limit of which was as low as $44 \mathrm{ppb}$ for NO2 and $262 \mathrm{ppb}$ for nitrotoluene. The recovery time of that sensor was $\sim 10 \mathrm{~h}$ due to the high bond energy between the CNT and some gases. Then this recovery time was reduced to 10 min by exposing to UV radiation which facilitated the desorption of gas molecules.

To reduce the sensor recovery time after gas detection by a sorption mechanism, attempts were made to accelerate gas desorption by heating sensor detectors [6].

The possibility of fabricating multiwalled CNT based sensors was discussed [7,8]. Sensor on the basis of ultrathin CNT films [9] was used for NO2 and NH3 detection at room temperature. The authors proposed a method of synthesizing $\sim 5 \mathrm{~nm}$ thick films with a high density of nanotubes ensuring high sensitivity and reproducibility of the sensor, i.e. $1 \mathrm{ppm}$ for $\mathrm{NO} 2$ and $7 \mathrm{ppm}$ for NH3. Gas desorption was accelerated by UV exposure.

CNT are often modified by adding the carboxyl group - $\mathrm{COOH}$. This group creates reactive sections at the terminations and the side walls of the CNT where active interaction with various compounds occurs. For example, it was shown [10] that sensors synthesized from carboxylated single-walled CNT were sensitive to CO with a 1 ppm detection limit whereas pure single-walled CNT did not respond to this gas. The NO2 gas sensitivity of single-walled CNT functionalized by the amino group $-\mathrm{NH} 2$ was studied [11]. The amino group acts as a charge transfer agent of the semiconducting CNT that increases the number of electrons transferred from the nanotube to the NO2 molecule.

Carbon nanotube boundary-modified carboxyl group was created. The authors used a multi-walled nanotube attached to the golden pyramid of the microscope's cantilever. A carboxyl group formed at the open nanotube boundary. It was reported that carboxylated $\mathrm{CNT}$ are sensitive to ethanol vapors and $\mathrm{NO}, \mathrm{CO}$ and $\mathrm{NO} 2$ gases.

If necessary the carboxyl group can be substituted for other functional groups using methods of organic chemistry [12]. Despite previous practical studies that have established the possibility of functionalization of CNT by some chemical groups, theoretical calculations of the possibility of interaction of the resulting system with different atoms and molecules have not been carried out, nor has the most preferred functional group been identified to determine alkali metal atoms. This determines the novelty of the study being conducted.

Thus, the studies we have carried out are relevant, as modeling the interaction of alkali metal atoms with the CNTfunctional group system may be the basis for the creation of an ultra-miniature highly sensitive sensor for determining the microvalues of the substance. Such a sensor can be used in medicine, to solve environmental needs, to determine residual traces of narcotic and psychotropic substances, etc.

\section{Formulation of the problem}

We studied the mechanism of carboxyl functional group attachment to a single-walled semi-infinite carbon nanotube zig-zag $(6,0)$. Nanotube was simulated within the molecular cluster model using the DFT calculation method. One of the cluster boundaries was closed by pseudoatoms hydrogen, and a carboxyl group was attached to the carbon atom at the other CNT boundary.

The mechanism of carboxyl group attachment to a selected carbon atom at an open nanotube boundary was simulated by step-by-step approximation of the carboxyl group with a $0.01 \mathrm{~nm}$ step along a perpendicular line to the tube boundary and oriented to a $\mathrm{C}$ atom. As a result the formation of a chemical bond between the nanotube and carboxyl group was observed (Fig. 1). 


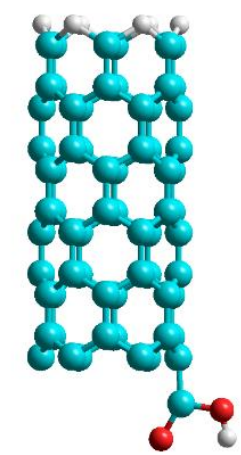

Fig. 1: Model of semi-infinite CNT $(6,0)$ with functional carboxyl group.

We studied the interaction mechanism between potassium, sodium and lithium atoms with terminal oxygen and hydrogen atoms of the carboxyl group. The process was simulated by step-by-step $(0,1 \AA$ ) approximation of the selected metal atoms to the $\mathrm{O}$ or $\mathrm{H}$ atom of the functional group (fig. 2). The figures show the characteristic minimums lying in the negative range of values, which may indicate the presence of interaction of the CNT-carboxyl group system with alkali metal atoms.

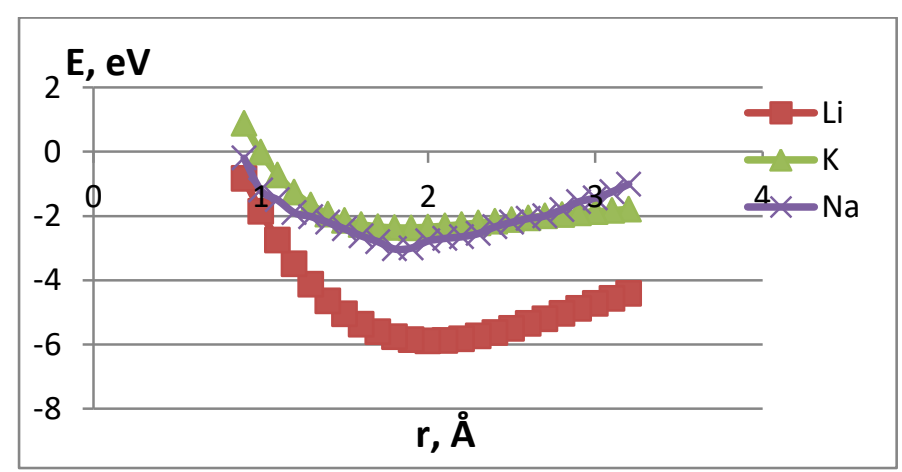

a)

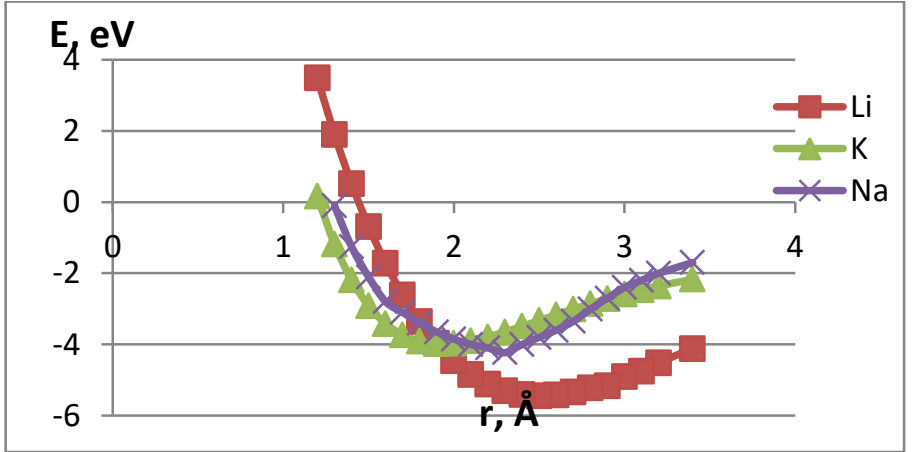

b)

Fig. 2: Energy profiles of interaction between $\mathrm{CNT}$ modified by carboxyl group $-\mathrm{COOH}$ and $\mathrm{Na}, \mathrm{K}$ and $\mathrm{Li}$ depending on distance between (a) metal atoms and hydrogen atom of the group and (b) metal atoms and oxygen atom of the group.

The main parameters of $\mathrm{K}, \mathrm{Li}$ and $\mathrm{Na}$ atom attachment to the atoms of the carboxyl group are in the Table 1 . The interaction distances corresponding to the minima in the energy profiles are quite large. So we can assume that the interaction between the functional group atoms and the selected metal atoms is the weak Vander-Waals one. This is an important result confirming the possibility of multiple reusing of these probes without destruction.

Table 1: Main parameters of $\mathrm{K}, \mathrm{Li}, \mathrm{Na}$ attachment to the $\mathrm{O}$ and $\mathrm{H}$ atoms of the carboxyl group.

\begin{tabular}{|c|c|c|}
\hline $\begin{array}{c}\text { Interatomic } \\
\text { bond }\end{array}$ & $\mathrm{r}_{\text {int }}, \AA$ & $\mathrm{E}_{\text {int }}, \mathrm{eV}$ \\
\hline $\mathrm{Na}-\mathrm{O}$ & 2.2 & -3.21 \\
\hline $\mathrm{Na}-\mathrm{H}$ & 1.8 & -1.77 \\
\hline $\mathrm{K}-\mathrm{O}$ & 2.5 & -4.3 \\
\hline
\end{tabular}




\begin{tabular}{|c|l|l|}
\hline K-H & 1.8 & -1.04 \\
\hline Li-O & 2.0 & -4.39 \\
\hline Li-H & 1.9 & -4.62 \\
\hline
\end{tabular}

Then we studied the scanning of a surface containing sodium, potassium or lithium atoms for the determination of the sensitivity of CNT with functional group to selected chemical elements. The process was simulated by step-by-step approximation of the metal atoms or ions to the functional group that was parallel to the modified nanotube boundary (Fig.

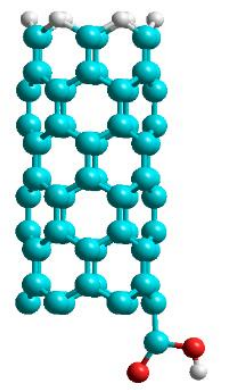

Fig. 3: Simulation of scanning of an arbitrary surface area containing Na atom (shown as purple ball). Dashed line shows sodium atom migration relative to nanotube with functional carboxyl group. Green balls in the figure show carbon atoms, red balls are oxygen atoms and white ones are hydrogen atoms.

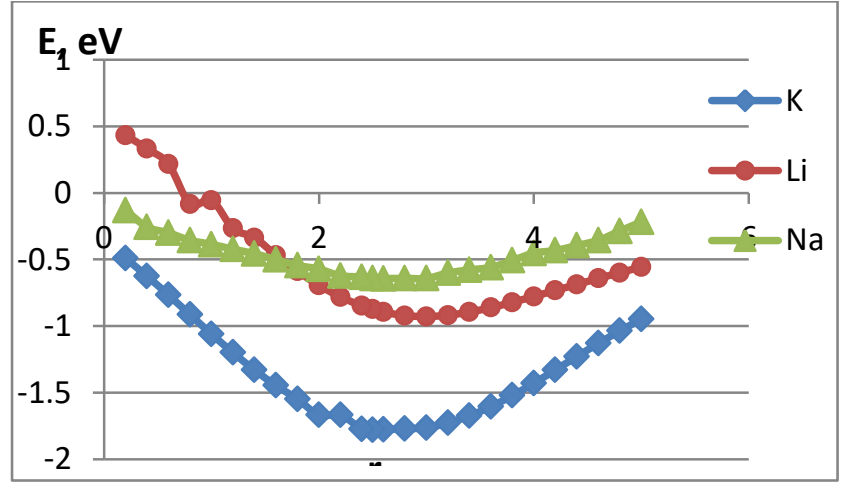

a)

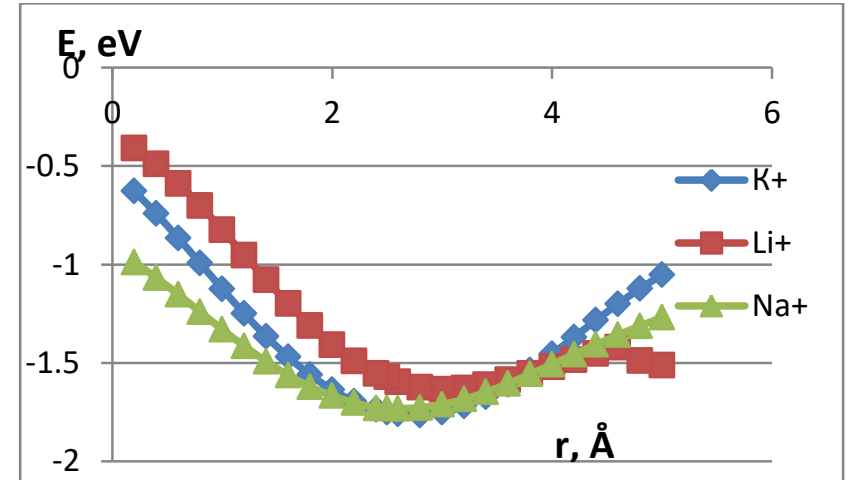

b)

Fig. 4: Profiles of energy interaction between (a) atoms or (b) ions of metal (K, Li, Na)and CNT $+\mathrm{COOH}$ system obtained by simulation of scanning. $r=0$ is the point under the hydrogen atom of the carboxyl group.

Analysis of the interaction energy profiles (Fig. 4) showed that the modified nanotube became sensitive to the selected metals. The energy profiles had typical minima. Main parameters of interaction are summarized in Table 2. The minimum energy values correspond to the position of the selected atom or ion under the oxygen atom of the carboxyl group. An 
interaction distance ranging from 3.0 to $2.5 \mathrm{~A}$ makes it possible to judge the presence of physical adsorption. Such interaction can predict the possibility of multiple use of a carboxylated CNT sensor. The results prove the possibility of using modified CNT as sensors for some elements and radicals. Their presence can be detected by controlling the change in the potential in the system.

Table 2: Main parameters of interaction of carboxylated CNT (6.0) with metal atoms and ions asdetermined by surface scanning.

\begin{tabular}{|c|c|c|}
\hline Atom/ion & $\mathrm{r}_{\text {int }}, \AA$ & $\mathrm{E}_{\text {int }}, \mathrm{eV}$ \\
\hline $\mathrm{Na}$ & 3.0 & -0.64 \\
\hline $\mathrm{Na}^{+}$ & 2.6 & -1.73 \\
\hline $\mathrm{K}$ & 2.5 & -1.77 \\
\hline $\mathrm{K}^{+}$ & 2.8 & -1.76 \\
\hline $\mathrm{Li}$ & 3.0 & -0.93 \\
\hline $\mathrm{Li}^{+}$ & 3.0 & -1.63 \\
\hline
\end{tabular}

To create a more complete picture and determine the most energy-efficient system for determining alkaline atoms, we studied the interaction of metal atoms and ions with carbon nanotubes modified amino and nitro group (Fig .5). The energy curves of the interaction are in the fig 6.

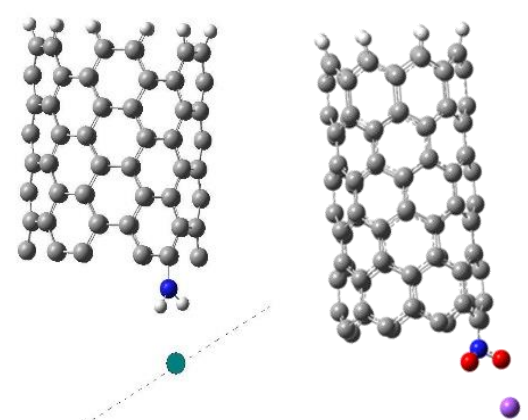

Fig. 5: Simulation of scanning of an arbitrary surface area containing Li and $\mathrm{Na}$ atom (shown as blue and purple ball).
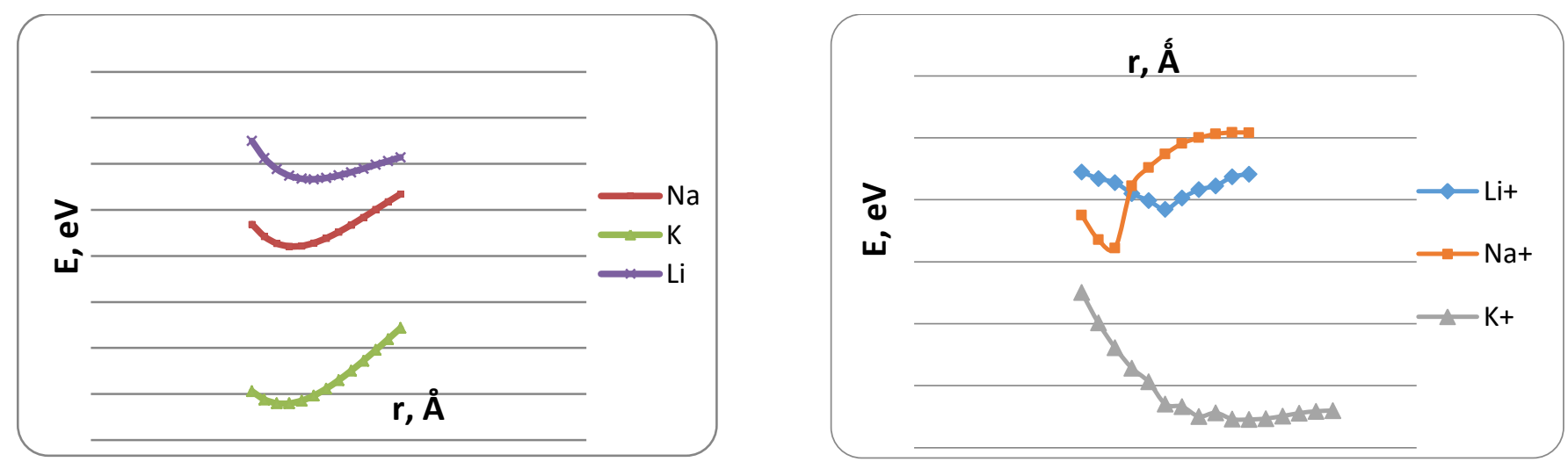

a) 

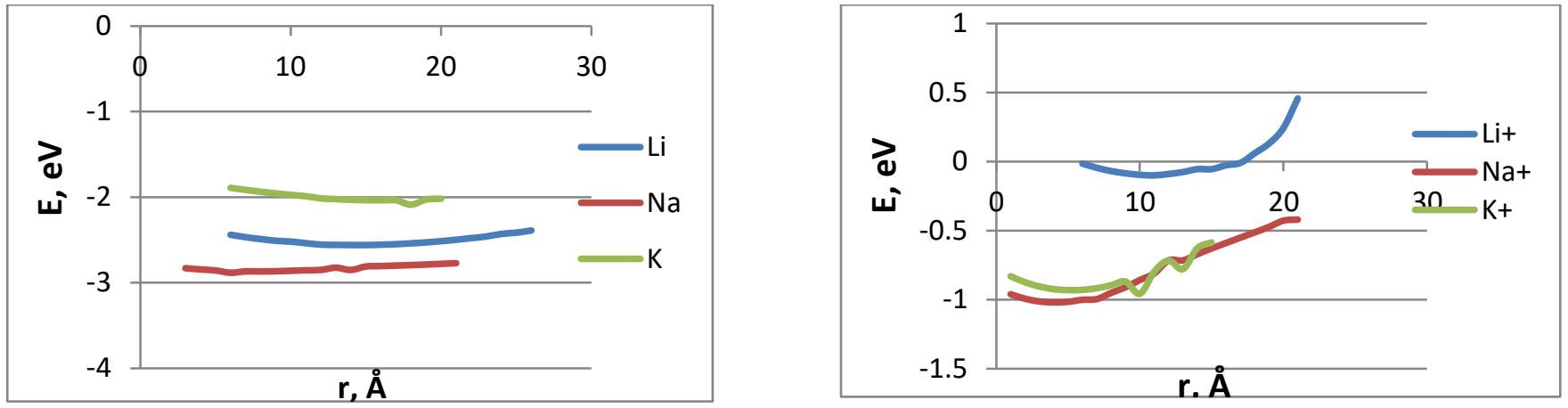

b)

Fig. 6: The energy curves of the interaction between atoms (or ions) of metals (Na, K, Li) and CNT (6, 6), modified amino (a) and nitro (b) group.

In the depicted potential enenrgy curves (Fig 6) there are visible minimums which, as in the case of the modified carboxyl group of CNT, lie in the negative range, which also makes it possible to judge the presence of interaction between nanosystems and potassium, lithium and sodium atoms. Main parameters of interaction are given in Tables 3, 4

Table 3: Main parameters of K, Li, Na attachment to CNT (6.0) modified by amino group.

\begin{tabular}{|c|c|c|}
\hline Atom/ion & $\mathrm{r}_{\text {int }}, \AA$ & $\mathrm{E}_{\text {int }}, \mathrm{eV}$ \\
\hline $\mathrm{Na}$ & 1.6 & -2.43 \\
\hline $\mathrm{K}$ & 1.6 & -3.22 \\
\hline $\mathrm{Li}$ & 1.8 & -1.0 \\
\hline $\mathrm{Na}$ & 1.2 & -3.21 \\
\hline $\mathrm{K}^{+}$ & 2.0 & -4.30 \\
\hline $\mathrm{Li}^{+}$ & 1.5 & -3.39 \\
\hline
\end{tabular}

Table 4: Main parameters of interaction of CNT (6.0) modified by amino group with K, Li, Na atoms and ions as determinated by arbitrary surface scanning simulation.

\begin{tabular}{|c|c|c|}
\hline Atom/ion & $\mathrm{r}_{\text {int }}, \AA$ & $\mathrm{E}_{\text {int }}, \mathrm{eV}$ \\
\hline $\mathrm{Na}$ & 2.0 & -5.21 \\
\hline $\mathrm{K}$ & 2.0 & -2.00 \\
\hline $\mathrm{Li}$ & 1.9 & -3.48 \\
\hline $\mathrm{Na}$ & 1.2 & -2.23 \\
\hline $\mathrm{K}^{+}$ & 1.4 & -5.15 \\
\hline $\mathrm{Li}^{+}$ & 1.5 & -2.36 \\
\hline
\end{tabular}

Some of the main parameters characterizing the scanning process are summarized in tables 1-4. All interaction energies in scanning simulation range from $0.5 \mathrm{eV}$ to $5.2 \mathrm{eV}$. This value of interaction energy indicates the presence of physical adsorption. Also, by analyzing the values of interaction energy and interaction distance, it is possible to conclude 
the most advantageous with respect to interaction with alkali metal atoms to the functional group. This information is discussed in the conclusion.

\section{Conclusion}

From the compare of the results, we concluded that the nanotubes functionalized by carboxyl, amino or nitro groups displayed chemical sensing the atoms and ions of the metals: potassium, sodium, and lithium. The most pronounced sensory response was observed for CNT functionalized with amino group. This sensor system displayed the highest energy of interaction with the atoms and ions of the considered alkali metals.

\section{Acknowledgements}

The reported research was funded by Russian Foundation for Basic Research and the government of Volgograd region, grant № 19-43-340005 r_a

The reported research was funded by Russian President's grant № 798.2019.1.

The reported research was funded by Russian President's grant № MK-1758.2020.8.

\section{References}

[1] M. S. Dresselhaus, G. Dresselhaus, P. Avouris, "Carbon Nanotubes: Synthesis, Structure, Properties, and Application," Springer-Verlag 2000.

[2] P. N. D'yachkov, "Electronic properties and applications of nanotubes," BINOM, Laboratoriya znanii, Moscow, 2010.

[3] W-D. Zhang, W-H. Zhang, "Carbon nanotubes as active components for gas sensors," J. Sens, 2009.

[4] N. P. Boroznina, I.V. Zaporotskova, L.V. Kozhitov, Y.N. Parhomenko, "Sensor properties of carbon nanotubes," News of universities, Materials electronic technology, 2017, vol. 19, no. 4, pp. 5-21.

[5] J. Li, Y. Lu, Q. Ye, M. Cinke, J. Han, M. Meyyappan, "Carbon nanotube sensors for gas and organic vapor detection," Nano Lett, vol. 7, pp. 929-933, 2003.

[6] H.-Q. Nguyen, J.-S. Huh, "Behavior of single-walled carbon nanotube-based gas sensors at various temperatures of treatment and operation," Sensors and Actuators B, vol. 117, no. 2, pp. 426-430, 2006.

[7] O. K. Varghese, P. D. Kichambre, D. Gong, K. G. Ong, E. C. Dickey, C. A. Grimes, "Gas sensing characteristics of multi-wall carbon nanotubes," Sens. Actuators B: Chem., vol. 81, no. 1, pp. 32-41, 2001.

[8] L. H. Nguyen, T. V. Phi, P. Q. Phan, H. N. Vu, C. Nguyen-Duc, "Fossard F. Synthesis of multi-walled carbon nanotubes for NH3 gas detection," Physica E, vol. 37, no. 1-2, pp. 54-57, 2007.

[9] C. Piloto, F. Mirri, E. A. Bengio, M. Notarianni, B. Gupta, M. Shafiei, M. Pasquali, N. Motta, "Room temperature gas sensing properties of ultrathin carbon nanotube films by surfactant-free dip coating," Sens. Actuators B: Chem., vol. 227, pp. 128-134, 2016.

[10] D. Fu, H. Lim, Y. Shi, X. Dong, S. G. Mhaisalkar, Y. Chen, S. Moochhala, L.-J. Li, "Differentiation of gas molecules using flexible and all-carbon nanotube devices," J. Phys. Chem. C, vol. 112, no. 3, pp. 650-653, 2008.

[11] T. H. Tran, J.-W. Lee, K. Lee, Y. D. Lee, B.-K. Ju, "The gas sensing properties of single-walled carbon nanotubes deposited on an aminosilane monolayer," Sens. Actuators B: Chem, vol. 129, no.1, pp. 67-71, 2008.

[12] J. Maklin, T. Mustonen, K. Kordas, S. Saukko, G.Toth, J. Vahakangas, "Nitric oxide gas sensors with functionalized carbon nanotubes,” Physica Status Solidi B, vol. 244, no. 11, pp. 4298-4302, 2007. 